\title{
Drift waves in helically symmetric stellarators
}

\author{
T. Rafiq and C. C. Hegna \\ Engineering Physics Department, University of Wisconsin, Madison, Wisconsin 53706-1609
}

(Received 21 July 2005; accepted 4 October 2005; published online 11 November 2005)

The local linear stability of electron drift waves and ion temperature gradient modes (ITG) is investigated in a quasihelically symmetric (QHS) stellarator and a conventional asymmetric (Mirror) stellarator. The geometric details of the different equilibria are emphasized. Eigenvalue equations for the models are derived using the ballooning mode formalism and solved numerically using a standard shooting technique in a fully three-dimensional stellarator configuration. While the eigenfunctions have a similar shape in both magnetic geometries, they are slightly more localized along the field line in the QHS case. The most unstable electron drift modes are strongly localized at the symmetry points (where stellarator symmetry is present) and in the regions where normal curvature is unfavorable and magnitude of the local magnetic shear and magnetic field is minimum. The presence of a large positive local magnetic shear in the bad curvature region is found to be destabilizing. Electron drift modes are found to be more affected by the normal curvature than by the geodesic curvature. The threshold of stability of the ITG modes in terms of $\eta_{i}$ is found to be $2 / 3$ in this fluid model consistent with the smallest threshold for toroidal geometry with adiabatic electrons. Optimization to favorable drift wave stability has small field line curvature, short connection lengths, the proper combination of geodesic curvature and local magnetic shear, large values of local magnetic shear, and the compression of flux surfaces in the unfavorable curvature region. (C) 2005 American Institute of Physics. [DOI: 10.1063/1.2130313]

\section{INTRODUCTION}

Drift wave instabilities have been extensively studied in axi-symmetric tokamaks using linear and non-linear drift wave theories. However, very limited work on these instabilities in fully three-dimensional stellarator geometries have been published (see, e.g., Refs. 1-9). This is due to its complicated three-dimensional structure and the related high resolution requirements for a numerical treatment. Furthermore, transport in conventional stellarators has been characterized by strong neoclassical levels, and anomalous transport has therefore had less importance. However, modern stellarators ${ }^{10,11}$ are designed to minimize neoclassical transport, potentially leading to anomalous transport originating from electrostatic turbulence as the primary cause of energy and particle losses. Stellarators are flexible devices allowing for a multitude of possible variations in the configurations. It is therefore of great interest to consider geometrical details on drift wave stability. The objective of this paper is to investigate the linear properties of drift waves using cold ion $(i \delta)^{12}$ and ion-temperature-gradient (ITG) models ${ }^{13-15}$ in helically symmetric stellarator with the purpose of contributing to the understanding of the geometrical effects on these instabilities.

A four-field period HSX (helical symmetric experiment) stellarator ${ }^{16}$ is selected to study the effect of geometrical properties such as local magnetic shear, magnetic field strength, normal curvature, geodesic curvature, plasma shaping, on the mode localization, mode structure, and stability. The Helically Symmetric Experiment (HSX) is a quasihelically symmetric stellarator experiment $(\mathrm{QHS})^{10,11}$ at the University of Wisconsin-Madison. HSX has a major radius of $1.2 \mathrm{~m}$ and a minor radius of $0.09-0.13 \mathrm{~m}$, depending on the machine configuration. It is a unique toroidal experiment that has an aspect ratio of eight, but a toroidal curvature close to zero. Hence it has a helical axis of symmetry closely approximate to the helical symmetry present in a straight stellarator. By restoring an axis of symmetry to a toroidal stellarator, the quasihelical stellarator solves one of the fundamental disadvantages of conventional stellarators; the poor neoclassical transport in the low collisionality regime. In addition to the modular coils that provide the base QHS configuration, there is an additional set of auxiliary coils that break the helically symmetry and allow the neoclassical transport and the stability limit to be independentally controlled. Specifically, operating HSX in the Mirror mode configuration increases the neoclassical transport and parallel viscous damping by orders of magnitude (back to the level of conventional stellarators) without appreciable changes in the rotational transform, magnetic well depth, or ideal local MHD stability limit. Neoclassical transport is optimized in QHS; it is likely that anomalous transport originating from electrostatic turbulence as the primary cause of energy and particle losses will become dominant. In particular, electron drift waves are likely to dominate in HSX plasma since ECRH heating produces plasma with $T_{e} / T_{i} \gg 1$. Hence, electron drift waves and the associated anomalous transport are emphasized. The influence of plasma geometry on linear drift waves is studied in the QHS configuration and the results are compared and contrasted with the results of a Mirror configuration which has high neoclassical transport. The important question to be discussed here is whether one stellarator geometry is favored over another or if an effective optimization is possible with regard to microinstabilities.

In the model for the electron drift waves, we assume a 
response close to adiabatic for the electrons and a cold ion response. The reason to consider this simple model is to emphasize the effect of geometry. Specifically, the role of local magnetic shear and magnetic field curvature on the structure and stability of the modes is examined. Linear stability of ITG modes is also studied using a two fluid reactive model that includes first order finite Larmor radius (FLR) effects as well as parallel ion dynamics and Boltzmann electrons. Both models are formulated in the ballooning representation ${ }^{17}$ and the drift wave problem is set as an eigenvalue equation along a magnetic field line. To solve the drift wave equations, we have used standard shooting technique and are applying Wentzel-Kramers-Brillouin (WKB) type boundary conditions.

In general, we have found that the most unstable modes are more localized along the field line. This is true for both geometries. While the eigenfunction in both geometries is similar, modes are found to be more localized in the QHS case than in the Mirror case. Additionally, the growth rate is found to be somewhat smaller in the Mirror case due to the slightly smaller bad curvature and large $|\mathbf{B}|$ in the Mirror geometry. The effect of the local shear of the magnetic field and local shaping of the neighboring flux surfaces is also found to be important. The existence of the high frequency modes, their localization along the field lines, and their frequencies and growth rates are found to be strongly dependent on the local magnetic shear and the normal curvature. However, modes are not appreciably affected by geodesic curvature. The edge is found to be more unstable as compared to center. This is due to the scaling of the field line bending which is large at the center and small at the edge.

The remainder of the paper is structured as follows. In Sec. II, the equilibrium magnetic field is specified in straight field line coordinates and the contravariant and covariant basis vectors are calculated. The equilibrium is computed using the variational moments equilibrium $(\mathrm{VMEC})^{18}$ code with fixed boundary conditions for QHS and Mirror configurations. In Sec. III and Sec. IV, the $i \delta$ and ITG models are used as physical models to describe the electron drift modes and the ion-temperature-gradient modes. In Sec. V the results and a discussion thereof are presented. Finally, a summary is given in Sec VI.

\section{THE MAGNETIC FIELD CONFIGURATION OF THE TOROIDAL SYSTEM}

The magnetic field configurations can be expressed in terms of the Boozer flux coordinates $(s, \theta, \zeta)$ where $\theta$ and $\zeta$ are generalized poloidal and toroidal angles and $s=2 \pi \psi / \psi_{p}$ is the normalized flux (radial) coordinate as given in Ref. 19. By construction, the radial label ranges from 0 (at the magnetic axis) to 1 (at the last closed magnetic surface). Here $2 \pi \psi$ is the poloidal magnetic flux bounded by the magnetic axis and the $\psi=$ const. surface and $\psi_{p}=\pi B_{0} \bar{a}^{2} / q$, the total poloidal magnetic flux. Here $B_{0}$ is the magnetic field at the axis, $\bar{a}$ is the average minor radius, $q=2 \pi / \iota$ is the safety factor, and $\iota=\iota(\psi)$ is the rotational transform which measures the helical twist of the magnetic field lines. The magnetic field $B$ is expressed as

$$
\mathbf{B}=\nabla \alpha \times \nabla \psi=\dot{\psi} \nabla \alpha \times \nabla s, \quad \text { with } \dot{\psi} \equiv \frac{d \psi}{d s}=\frac{B_{0} \bar{a}^{2}}{2 q},
$$

which fulfills $\boldsymbol{\nabla} \cdot B=0$ and $B \cdot \nabla \psi=B \cdot \nabla \alpha=0$, implying that $\psi$ and $\alpha$ are stream functions (constant along a field line) of the magnetic field. It further implies that $\psi=$ const. is a magnetic flux surface and that $\alpha=\zeta-q \theta$ is a field line label on this surface. A given magnetic flux surface can be represented by a square cell $0 \leqslant \theta<2 \pi, 0 \leqslant \zeta<2 \pi$ with the edges $\theta$ $=0,2 \pi$ and $\zeta=0,2 \pi$ topologically identified. While the equilibrium is periodic in $\zeta$ and $\theta$, for the eigenvalue problem using ballooning formalism, described in the next section, the magnetic field lines lie in the domain $-\infty<\theta<\infty$, $-\infty<\zeta<\infty .{ }^{20}$ The equilibrium code VMEC solves the MHD force balance equation

$$
\mathbf{J} \times \mathbf{B}=\boldsymbol{\nabla} P,
$$

in three-dimensional geometry. Here $J=\mu_{0} \nabla \times B$ is the plasma current, $\mu_{0}$ is the permeability of free space, and $P$ is the isotropic plasma pressure. In VMEC, two flux surface quantities are prescribed. One is the net toroidal plasma current enclosed within each flux tube and the second is the plasma pressure profile. In this paper we use the same plasma pressure profile for all VMEC equilibria. The equilibria for the QHS and the Mirror configurations are computed with fixed boundary conditions and for a set of 98 magnetic surfaces. The VMEC code uses a coordinate system that has been optimized by minimizing the number of harmonics required to represent the equilibrium quantities so that the equilibrium calculation is very efficient. However, the VMEC coordinate system is not a straight field line coordinate system. Thus, before solving the drift wave equation, we first transform the equilibrium to Boozer coordinates. The VMEC code uses an inverse equilibrium representation, in which the real space cylindrical coordinates are given by Fourier expansions. The Boozer system and the standard cylindrical coordinates $\left(R, \phi_{c}, z\right)$ are related through the Fourier series (for a given magnetic surface, $s$ = constant)

$$
\begin{aligned}
& R=\sum_{m=0}^{n_{p}} \sum_{n=-n_{t}}^{n_{t}} R_{m n}(s) \cos (m \theta+n N \zeta), \\
& \phi_{c}=\zeta-\frac{2 \pi}{N} \sum_{m=0}^{n_{p}} \sum_{n=-n_{t}}^{n_{t}} \phi_{m n}(s) \sin (m \theta+n N \zeta), \\
& z=\sum_{m=0}^{n_{p}} \sum_{n=-n_{t}}^{n_{t}} z_{m n}(s) \sin (m \theta+n N \zeta) .
\end{aligned}
$$

Here $n_{p}$ and $n_{t}$ are the maximum poloidal and toroidal Fourier components and are both input parameters of VMEC, and $N$ is the number of field period, which is different for different fusion machines. In this case, we use $n_{p}$ $=12, n_{t}=8$, and $N=4$. The Fourier coefficients $R_{m n}, \phi_{m n}$, and $z_{m n}$, and the rotational transform $\iota$ are calculated as function of the flux coordinate $s$ by the VMEC $\operatorname{code}^{18}$ and then mapped over to the Boozer coordinate system $(s, \theta, \zeta) .^{21,22}$ 
The position vector $r_{p}$ of any point $(s, \theta, \zeta)$ at a flux surface $s$ in the coordinate system $(x, y, z)$ is $r_{p}$ $=\left(R \cos \phi_{c}, R \sin \phi_{c}, z\right)$ where the covariant basis vectors in the Boozer coordinate system are

$$
\mathbf{e}_{s}=\frac{\partial \mathbf{r}_{p}}{\partial s}, \quad \mathbf{e}_{\theta}=\frac{\partial \mathbf{r}_{p}}{\partial \theta}, \quad \mathbf{e}_{\zeta}=\frac{\partial \mathbf{r}_{p}}{\partial \zeta},
$$

and the associated contravariant basis vectors

$$
\begin{aligned}
& \boldsymbol{\nabla} s \equiv \mathbf{e}^{s}=\frac{\mathbf{e}_{\theta} \times \mathbf{e}_{\zeta}}{J}, \quad \boldsymbol{\nabla} \theta \equiv \mathbf{e}^{\theta}=\frac{\mathbf{e}_{\zeta} \times \mathbf{e}_{s}}{J}, \\
& \boldsymbol{\nabla} \zeta \equiv \mathbf{e}^{\zeta}=\frac{\mathbf{e}_{s} \times \mathbf{e}_{\theta}}{J} .
\end{aligned}
$$

The covariant basis vector $e_{\theta}$ is tangent to the $\theta$ coordinate curves, which lies at the intersection of the coordinate surfaces $s=$ constant and $\zeta=$ constant. The contravariant basis vector $\nabla \theta$ is perpendicular to the coordinate surface $\theta$ $=$ constant. The Jacobian (the determinant of the matrix produced by nine partial derivatives) of the transformation $J$ can be calculated using the set of covariant basis vectors

$$
J \equiv \mathbf{e}_{s} \cdot \mathbf{e}_{\theta} \times \mathbf{e}_{\zeta}=\frac{\dot{\psi}}{B^{2}}\left(B_{\theta}+q B_{\zeta}\right),
$$

where $B_{\theta}$ and $B_{\zeta}$, the covariant components of $\mathrm{B}$, are surface quantities, i.e., $B_{\theta}=B_{\theta}(s)$ and $B_{\zeta}=B_{\zeta}(s)$. Having calculated the derivatives of the cylindrical coordinates $R, \phi_{c}$, and $z$ with respect to $s$ numerically, Eq. (6) can be used to calculate derivatives of $B$ with respect to $\theta$ and $\zeta$ analytically, while the derivatives with respect to $s$ are calculated numerically. Using the reciprocal relation of contravariant and covariant basis vectors, Eq. (1) can be written as $B=\dot{\psi} / J\left(\mathbf{e}_{\theta}+q \mathbf{e}_{\zeta}\right)$ and the parallel gradient operator $e_{\|} \cdot \nabla$ (where $e_{\|}=B / B$ ) can be written

$$
\mathbf{e}_{\|} \cdot \boldsymbol{\nabla}=\frac{\dot{\psi}}{J B}\left(\frac{\partial}{\partial \theta}+q \frac{\partial}{\partial \zeta}\right)=\left.\frac{\dot{\psi} q}{J B} \frac{d}{d \zeta}\right|_{\text {field line }},
$$

using Eq. (6), we write

$$
\begin{aligned}
\boldsymbol{\nabla} \ln B= & \frac{1}{2}\left[\left(\frac{d}{d s} \ln \left(B_{\theta}+q B_{\zeta}\right)-\frac{\partial \ln J}{\partial s}\right) \boldsymbol{\nabla} s-\frac{\partial \ln J}{\partial \theta} \boldsymbol{\nabla} \theta\right. \\
& \left.-\frac{\partial \ln J}{\partial \zeta} \boldsymbol{\nabla} \zeta\right],
\end{aligned}
$$

and

$$
\frac{\partial J}{\partial s}=\frac{\partial \mathbf{e}_{s}}{\partial s} \cdot \mathbf{e}_{\theta} \times \mathbf{e}_{\zeta}+\mathbf{e}_{s} \cdot \frac{\partial \mathbf{e}_{\theta}}{\partial s} \times \mathbf{e}_{\zeta}+\mathbf{e}_{s} \cdot \mathbf{e}_{\theta} \times \frac{\partial \mathbf{e}_{\zeta}}{\partial s} .
$$

The field line curvature vector $\boldsymbol{\kappa}$ is

$$
\begin{aligned}
\boldsymbol{\kappa} \equiv & \mathbf{e}_{\|} \cdot \nabla \mathbf{e}_{\|}=q\left(\frac{\dot{\psi}}{J B}\right)^{2}\left[\frac{d}{d \zeta}\left(\mathbf{e}_{\theta}+q \mathbf{e}_{\zeta}\right)\right. \\
& \left.-\frac{1}{2} \frac{d \ln J}{d \zeta}\left(\mathbf{e}_{\theta}+q \mathbf{e}_{\zeta}\right)\right]
\end{aligned}
$$

or it can also be written in terms of metric elements as

$$
\boldsymbol{\kappa}=\frac{\kappa_{n}}{\sqrt{g^{s s}}} \nabla s+\frac{\kappa_{g}}{\sqrt{g^{s s}}}\left(\frac{\dot{\psi} g^{s s}}{B}\right)(\nabla \alpha-\wedge \nabla s),
$$

where $\wedge=g^{s \alpha} / g^{s s}$ is the local magnetic shear integrated along the field line (ILMS) as given in Ref. 23 and $g^{i j}=\boldsymbol{\nabla} i \cdot \nabla j$ is the dot product of the flux coordinates. When the curve under consideration lies within a surface, it is customary to define curvature components with respect to the surface. The component of $\kappa$ normal to the surface is called the normal curvature $\kappa_{n}$, whereas the component of $\kappa$, tangent to the surface, is referred to as the geodesic curvature, $\kappa_{g}$. The normal and geodesic components of $\boldsymbol{\kappa}$ are

$$
\kappa_{n}=\kappa \cdot \frac{\nabla s}{|\nabla s|}, \quad \kappa_{g}=\kappa \cdot\left(\frac{\nabla s}{|\nabla s|} \times \mathbf{e}_{\|}\right) .
$$

Regions of the configurations with a negative normal curvature are expected to be unstable to local pressure driven instabilities, whereas positive regions are expected to be stable. The local magnetic shear is the local rate of rotation of the magnetic field direction and is another equilibrium quantity that plays an important role in the stability as given in Refs. $24-30$ and can be written as ${ }^{12}$

$$
\begin{aligned}
S & =\left(\hat{s} \times \mathbf{e}_{\|}\right) \cdot \boldsymbol{\nabla} \times\left(\hat{s} \times \mathbf{e}_{\|}\right)=\left(\mathbf{e}_{\|} \cdot \boldsymbol{\nabla}\right) \wedge \\
& =\frac{\dot{\psi}^{2}|\boldsymbol{\nabla} s|^{2}}{J B^{2}}\left[-\frac{d q}{d s}+\left(\frac{\partial}{\partial \theta}+q \frac{\partial}{\partial \zeta}\right)\left\{\frac{(\boldsymbol{\nabla} \zeta-q \boldsymbol{\nabla} \theta) \cdot \boldsymbol{\nabla} s}{|\boldsymbol{\nabla} s|^{2}}\right\}\right],
\end{aligned}
$$

where $\hat{s} \equiv \nabla s /|\nabla s|$ is a unit vector normal to the magnetic surface and pointing outwards.

\section{THE DRIFT WAVE MODELS}

A simple drift wave equation for a low- $\beta$ plasma, in which the electrons response is close to adiabatic, is derived by employing the $i \delta$ model. A small nonadiabaticity is included in the quasi-neutrality condition $n_{i} \approx n_{e}=(1$ $+i \delta) n_{0} e \phi / T_{e}$, which can be due to collisions, wave-particle resonance, dissipation due to electron trapping, or any other mechanism preventing electrons from freely moving along the field line. In the approach followed here, the mechanism represented by $\delta$ is independent of the position on the magnetic surface and is a free parameter. A conventional drift wave equation is derived leading to an ordinary differential equation along the field line by employing the WKB assumption in the coordinates $(\psi, \alpha, \zeta)$ and by using the standard ballooning mode formalism, $\hat{\phi}=\Phi(\zeta) \exp \left[-i \epsilon^{-1} S(\psi, \alpha)\right]$. The resulting eigenvalue equation is written as given in Ref. ${ }^{12}$ in the following form:

$$
\frac{d^{2} \Phi}{d \zeta^{2}}+U(\zeta, \Omega) \Phi=0
$$

where $\Phi(\zeta)$ is the eigenfunction and $U(\zeta, \Omega)$ is the effective potential given as 

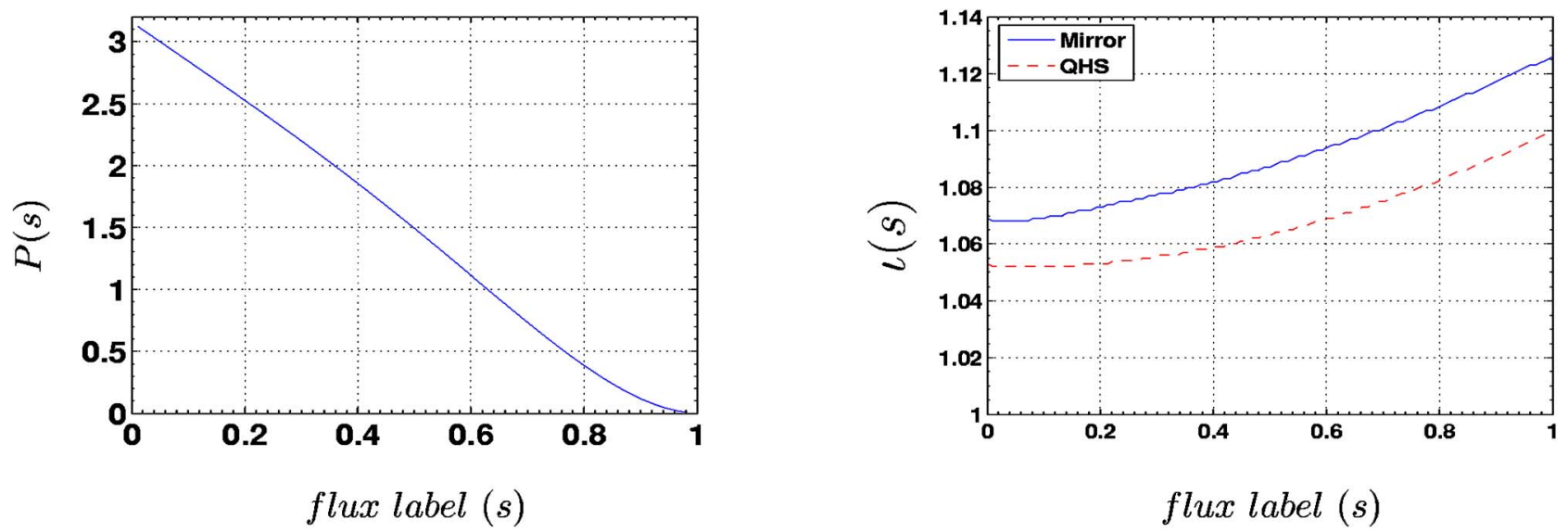

FIG. 1. (Color online). The variation of VMEC input plasma pressure profile, $P(s)$ (left), used in the calculation of magnetic field configurations and the VMEC output rotational transform, $\iota(s)$ (right), as a function of flux label (s).

$$
\begin{aligned}
U(\zeta, \Omega)= & -\left(\frac{J B}{q \bar{R} \dot{\psi}}\right)^{2}\left[\left(\Omega_{*}+\Omega_{d}\right) \chi \Omega\right. \\
& \left.-\left(1+\left(\frac{\chi B_{0}}{B}\right)^{2}\left(\hat{\mathbf{k}}_{\perp} \cdot \hat{\mathbf{k}}_{\perp}\right)+i \delta\right) \Omega^{2}\right] .
\end{aligned}
$$

Here,

$$
\begin{aligned}
& \Omega_{*}=\Omega_{*}(s)=-\frac{2}{\epsilon_{n} \bar{a}}, \quad \epsilon_{n}=\frac{L_{n}}{\bar{R}}, \quad \Omega=\frac{\bar{R} \omega}{c_{s}}, \\
& \chi=\epsilon^{-1} \frac{q \rho_{s o}}{\bar{a}} \frac{\partial S}{\partial \alpha}, \quad c_{s}=\sqrt{\frac{T_{e}}{m_{i}}}, \quad \hat{\phi} \equiv \frac{e \phi}{T_{e}}, \\
& \Omega_{d}=\Omega_{d}(s, \alpha, \zeta)=B_{0} \bar{R}\left(\frac{\mathbf{B} \times(\boldsymbol{\kappa}+\nabla \ln B)}{B^{2}}\right) \cdot \hat{\mathbf{k}}_{\perp}, \\
& \rho_{s o}=\frac{c_{s}}{e B / m_{i}},
\end{aligned}
$$

where $\epsilon$ is the WKB expansion parameter, $S$ is the ballooning mode eikonal, $L_{n}$ is the density scale length, $T_{e}$ is the electron temperature, and $c_{s}$ is the ion sound speed with electron temperature.

The perpendicular wave number is given as

$$
\begin{gathered}
\mathbf{k}_{\perp}=\epsilon^{-1} \frac{\partial S}{\partial \alpha}\left\{\boldsymbol{\nabla} \alpha+\Theta_{k} \dot{q} \boldsymbol{\nabla} s\right\}=\epsilon^{-1} \frac{q}{\bar{a}} \frac{\partial S}{\partial \alpha} \hat{\mathbf{k}}_{\perp} \\
\text { with } \Theta_{k}=\frac{\partial S}{\partial q} / \frac{\partial S}{\partial \alpha},
\end{gathered}
$$

where the normalized perpendicular wave vector is

$$
\begin{aligned}
\hat{\mathbf{k}}_{\perp} & =\hat{\mathbf{k}}_{\perp}\left(s, \alpha, \zeta, \theta_{k}\right) \\
& =\frac{\bar{a}}{q}\left[\boldsymbol{\nabla} \zeta-q \boldsymbol{\nabla} \theta-\left(\frac{\zeta-\zeta_{0}}{q}-\theta_{k}\right) \dot{q} \boldsymbol{\nabla} s\right]
\end{aligned}
$$

with $\theta_{k}=\Theta_{k}-\theta_{0}$ and $\dot{q}=d q / d s$. Equation (14) describes the universal electron drift modes in the presence of a dissipative mechanism. The correlation between the growth rate and local geometry can be clarified and analyzed in rewriting $\Omega_{d}$ and the normalized perpendicular wave vector $b$ as

$$
\begin{aligned}
\Omega_{d} & =-2 \chi \frac{\bar{R}}{\bar{a}}\left\{\frac{\kappa_{n}}{\sqrt{g^{s s}}}-\frac{\kappa_{g}}{\sqrt{g^{s s}}} \frac{B_{o}}{B} \chi_{g}\left(\wedge+\theta_{k} \dot{q}\right)\right\}, \\
b & =\chi_{\perp}^{2} \hat{\mathbf{k}}_{\perp} \cdot \hat{\mathbf{k}}_{\zeta=\zeta_{0}} \\
& =2 \frac{\chi^{2}}{q \chi_{g}}\left\{1+\left(B_{o} / B\right)^{2} \chi_{g}{ }^{2}\left\{\wedge^{2}+2 \theta_{k} \dot{q} \wedge+\left(\theta_{k} \dot{q}\right)^{2}\right\}\right\},
\end{aligned}
$$

where $\chi_{g} \equiv \bar{a}^{2} g^{s s} / 2 q$.

As discussed in the work of Dewar and Glasser ${ }^{20}$ the growth rate depends on the flux surface, the magnetic field line label, and the radial wave number. In principle one sets these three parameters and solves for the spectrum. However, in practice the eigenmode spectrum associated with a specific set of these three parameters is difficult to construct since the field line in general is infinitely long passing arbitrarily close to every point on a magnetic surface. In lieu of this issue, a shooting method is employed. With the shooting method used here, that part of the spectrum localized around the matching points is emphasized where the continuity of the eigenmodes is enforced. Hence by moving the matching point around one therefore recovers the complete spectrum. One can either do this by keeping the field line fixed and move the matching point on the field line or by moving from field line to field line. Formally these two methods are not identical as in the latter case the three parameters are not held fixed. However since every field line, on a nonrational surface, passes arbitrarily close to every point on the surface, the two spectra obtained are identical in the limit of arbitrary fine resolution. That both methods work in practice, and give the same results, can easily be demonstrated numerically as well. What is done here is slightly more advanced. Since the growth rate has a maximum at $\theta_{k}=0, \theta_{k}$ is redefined in such a way that it sets the radial mode number to zero at each matching point and the most unstable modes on the magnetic surface are picked up. In practice a scan of the $\theta_{k}$ is not calculated since the dependence of the eigenmodes with $\theta_{k}$ is very weak. 


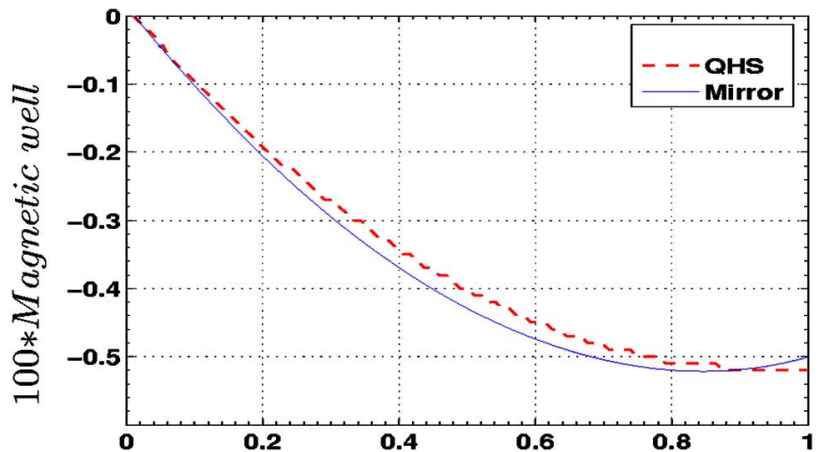

flux label $(s)$

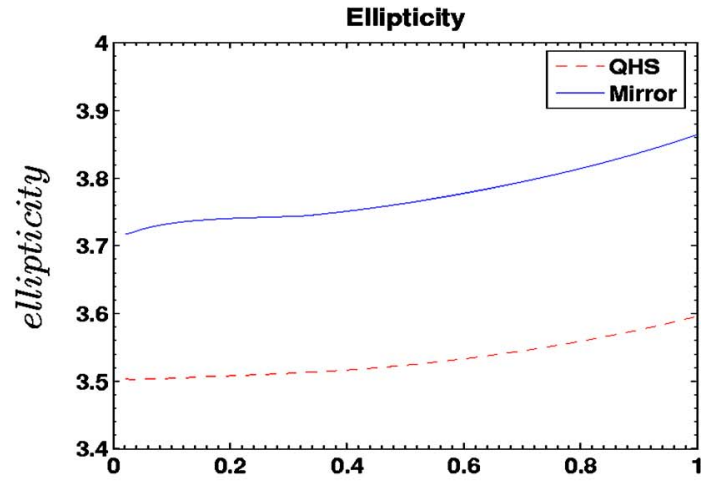

flux label $(s)$

FIG. 2. (Color online). Magnetic well depth (left) and ellipticity (right) as a function of flux label (s).

\section{ITG MODEL}

To investigate the ITG instability an advanced fluid model is used, which is derived in the short wavelength region taking Boltzmann electrons and using ion continuity, energy, and parallel momentum equations. The eigenmode equation as given in Refs. 14 and 15 derived by applying WKB type assumptions in Boozer coordinates can be written as

$$
\begin{aligned}
\frac{d^{2} \Psi}{d \zeta^{2}} & +\left(\frac{J B}{q \bar{R} \dot{\psi}}\right)^{2}\left[H^{-1}\left(\Omega-\frac{2 \chi}{\epsilon_{n} \bar{a}}\right)+\Omega\left(\frac{\chi B_{0}}{B}\right)^{2}\left(\hat{\mathbf{k}}_{\perp} \cdot \hat{\mathbf{k}}_{\perp}\right)\right. \\
& \left.-\chi \Omega_{d}\right] \Omega \Psi=0
\end{aligned}
$$

where $\Omega=\bar{R} \omega / C_{s}$, and $\Psi=H \Phi, \epsilon_{n}=L_{n} / \bar{R}, L_{n}^{-1}=-d \ln n_{o} / d s$,

$$
H=1+\tau^{-1}+\frac{\tau^{-1}}{\Omega-(5 / 3 \tau) \chi \Omega_{d}}\left[(2 / 3) \Omega+\frac{2 \chi}{\epsilon_{n} \bar{a}}\left(\eta_{i}-2 / 3\right)\right] \text {. }
$$

The physical problem is now reduced to an eigenvalue equation along the field line, which is similar to the problem

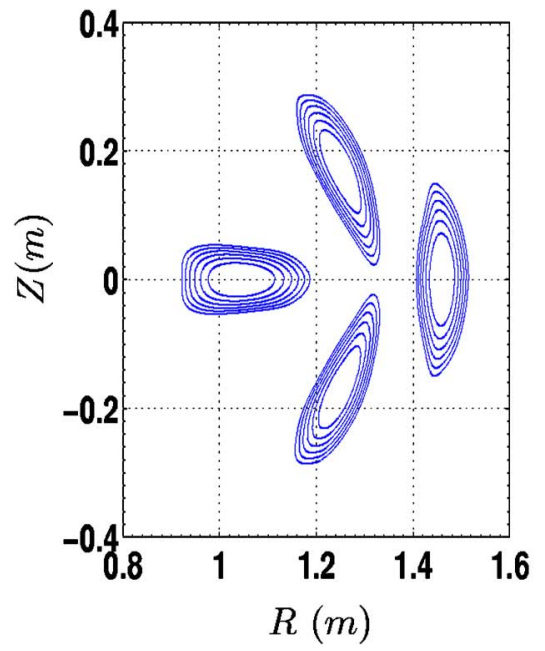

of a particle in a potential well in quantum mechanics. Hence, Eqs. (14) and (19) are solved numerically along a magnetic field line $(\zeta)$ on a given flux surface $(s)$. This is solved by applying appropriate boundary condition for large $|\zeta|$ and by demanding continuity of the eigenfunction and its first derivative at a point $\zeta=\zeta_{o}$, usually referred to as a "matching point." Hence, at a given magnetic surface $s$, the eigenfunction $\Phi$ and the corresponding normalized eigenfrequency $\Omega$ can be determined for given values of the equilibrium plasma density scale length $L_{n}$, and the free parameters $\chi$ and $\theta_{k}$. The former controls the magnitude of the perpendicular wave vector $k_{\perp}$ and the latter its orientation.

\section{NUMERICAL RESULTS AND DISCUSSION}

The drift wave equations are solved by a shooting algorithm using a sixth-order Numerov scheme and WKB type boundary conditions and by demanding continuity of the function at its first derivative at a matching point. Details of the boundary conditions and the numerical method used are given in Refs. 12 and 31.

The input pressure profile used in the calculation of the

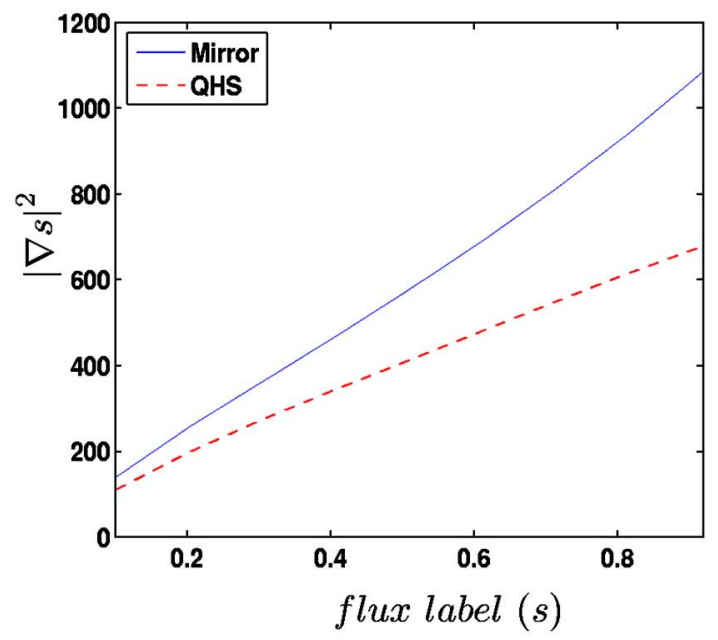

FIG. 3. (Color online). Cross sections in the QHS case at $\zeta_{o}=0, \zeta_{o}=1 / 4, \zeta_{o}=1 / 2$, and $\zeta_{0}=3 / 4$ (left), and the geometric quantity $|\nabla s|^{2}$ (right), for the Mirror and the QHS case. 


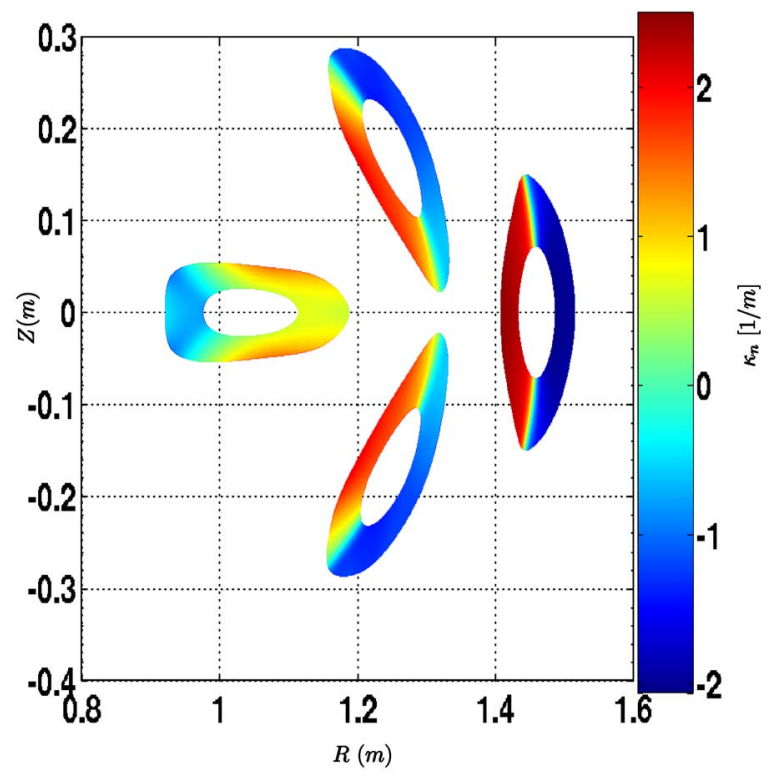

FIG. 4. (Color online). Regions of good and bad curvature in the base QHS configuration at $\zeta_{0}=0, \zeta_{0}=1 / 4, \zeta_{0}=1 / 2$, and $\zeta_{0}=3 / 4$ cross sections. The negative value corresponds to the bad curvature region.

magnetic field configurations of the QHS and the Mirror case are shown in Fig. 1 together with the $\iota$-profile which is an output from the code. The rotational transform $\iota$ in the QHS configuration varies from 1.05 at the center to 1.10 at the edge, while the transform for the Mirror configuration is very similar, going from 1.07 at the center to 1.13 at the edge. The well depth and ellipticity is plotted in Fig. 2 for the QHS and the Mirror modes of operation. In spite of very different neoclassical transport, the two configurations have roughly the same magnetic well depth around $5 \%$, with a slight hill toward the plasma edge. However, the Mirror equilibrium is more elliptic. The stabilization is expected for an elongated equilibrium due to the reduction of magnetic drift frequency.

The cross sections of the QHS configuration are provided in Fig. 3 (left). For the sake of clarity only 6 of the nested magnetic surfaces are plotted at $\zeta_{o}=0.0, \zeta_{o}=1 / 4, \zeta_{o}$ $=1 / 2$, and $\zeta_{o}=3 / 4$. The full equilibrium has been determined with 98 magnetic surfaces. The average major radius $(\bar{R})$ is $1.2 \mathrm{~m}$, the magnetic field strength at the magnetic axis is 0.3 $\mathrm{T}$ and the aspect ratio is $\bar{R} / \bar{a}=8.6(\bar{a}$ is the average minor

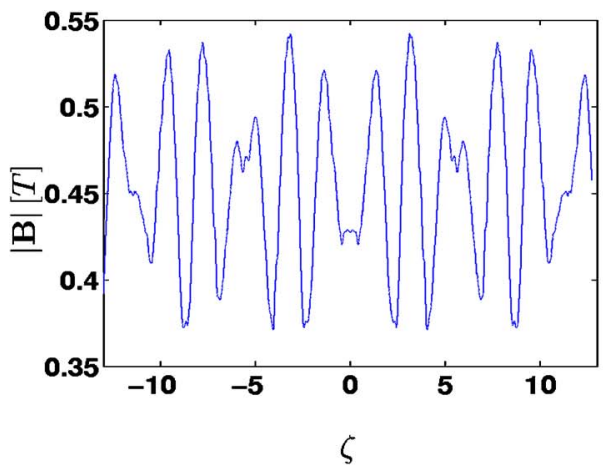

radius). The flux surface shape quantity $|\nabla s|^{2}$ for the QHS and the Mirror configurations are plotted in Fig. 3 (right). The flux surfaces are more compressed in the Mirror case than the QHS configuration.

Regions of good and bad curvature in one field period of the QHS at $\zeta_{o}=0.0, \zeta_{o}=1 / 4, \zeta_{o}=1 / 2$, and $\zeta_{o}=3 / 4$ are shown in Fig. 4. Unlike tokamaks, the regions of good and bad curvature rotate as a function of toroidal angle in QHS. At $\zeta_{o}=0.0$ the good curvature region is on the inside of the surfaces and bad curvature is on the outside as in tokamak or conventional stellarator. At $\zeta_{o}=1 / 4$ the region of good curvature has moved to the bottom of the device, while, at $\zeta_{o}$ $=1 / 2$ the good curvature has moved to the outside of the device. Finally, at $\zeta_{o}=3 / 4$ the good curvature region has moved to the top of the flux surfaces.

Figure 5 shows the variation in magnitude of the magnetic field strength as a function of $\zeta$ in the QHS (right) and in the Mirror configuration (left) on the magnetic surface $s$ $=0.8980$ along the magnetic field line specified by $\alpha=0$ (this field line passes through the point $\zeta_{o}=0.0$ and $\theta_{o}=0.0$ ).

The normalized normal curvature (left) and geodesic curvature (right) in both configurations as a function of $\zeta$ are shown in Fig. 6. Helical ripples, which decrease the connection length between good and bad curvature region, are evident. Around $\zeta=0.0$, slightly more bad normal curvature is found in the QHS case. Therefore, in the QHS case, a relatively higher growth rate is expected for highly localized modes along the field line. However, the structure of the geodesic curvature is similar in both geometries.

The local magnetic shear $S$ (left) and the field line bending term $\hat{k}_{\perp}^{2}$ (right) are shown in Fig. 7. A more negative local magnetic shear region along the field line is found in the Mirror case on this specific field line. Therefore the envelope of the eigenfunction in the QHS case is expected to be more localized than in the Mirror case. However, the growth rate is expected to be the same in both configurations for the modes localized in the region of $\zeta= \pm 1.0 \mathrm{rad}$ due to the similar values of the local magnetic shear in that region.

Figures 8(a) and 8(b) show the drift wave spectra calculated for the QHS and the Mirror configuration using the cold ion model on the flux surface $s=0.8980$ using the field line $\left(\theta_{o}=0, \zeta_{o}=0\right)$. Here the local magnetic shear is small, normal curvature is large negative (unfavorable), and geode-

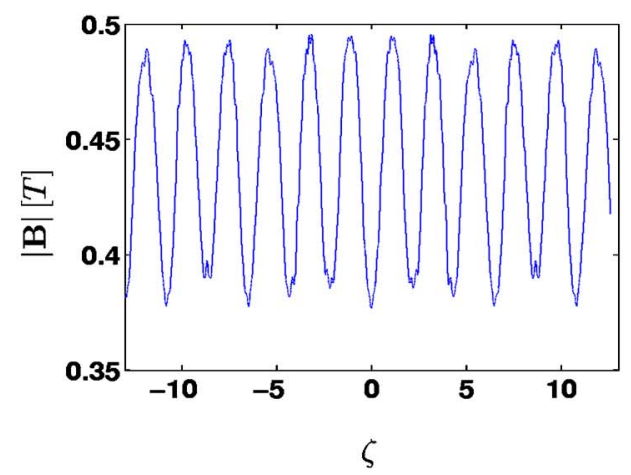

FIG. 5. (Color online). Variation of $|\mathbf{B}|$ in the Mirror case (left) and in the QHS case (right) along the magnetic field line with $\theta_{0}=0$, $\zeta_{0}=0$ on the magnetic surface $s=0.8980$. 

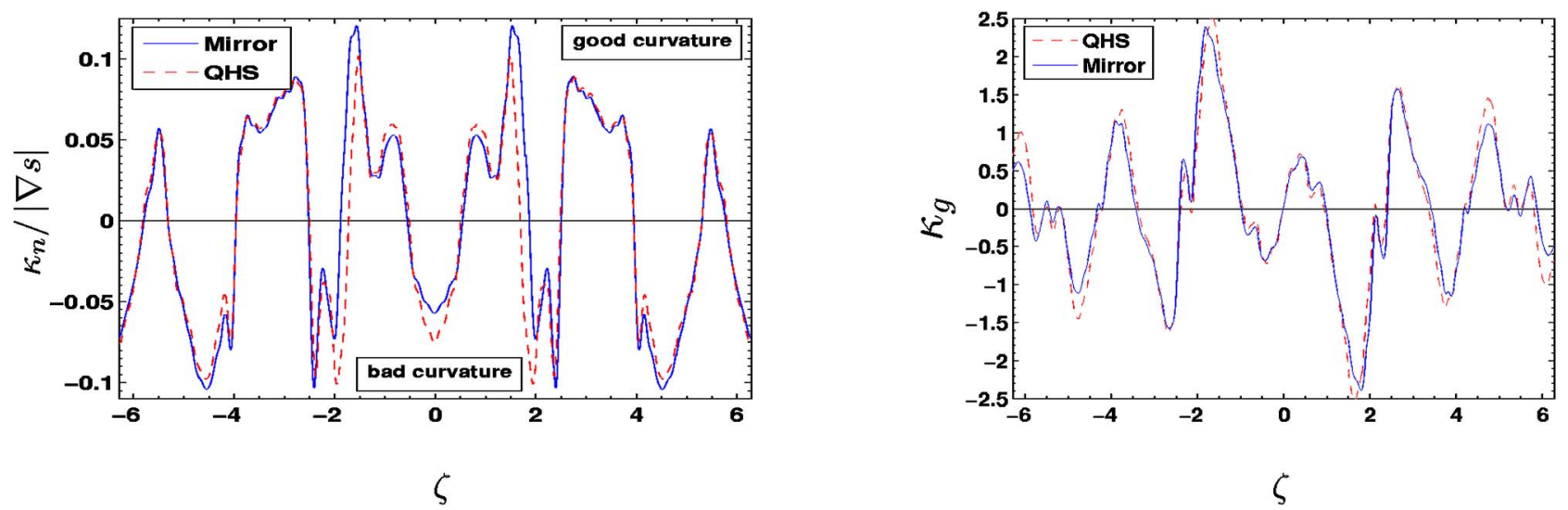

FIG. 6. (Color online). Variation of normalized normal curvature, $\kappa_{n} /|\nabla s|$ (left), and geodesic curvature, $\kappa_{g}(\zeta)$ (right), along the magnetic field line for the same parameters values as in Fig. 5.

sic curvature is zero. The parameter values used are $b$ $=\left(k_{\perp} \rho_{s}\right)^{2}=\chi^{2}\left|\hat{k}_{\perp} \cdot \hat{k}_{\perp}\right|_{\zeta=\zeta_{o}}=\left(\epsilon^{-1} \partial s / \partial \alpha\right)^{2}|\nabla \alpha \cdot \nabla \alpha|_{\zeta=\zeta_{o}}=0.1$, and $\theta_{k}=0, \epsilon_{n}=L_{n} / \bar{R}=0.0417$, where $L_{n}$ is the radial density scale length, and $L_{n}^{-1}=-d \ln n_{0} /\left.d s \hat{s} \cdot \nabla s\right|_{\zeta=\zeta_{o}}$, where $\hat{s} \equiv \nabla s /|\nabla s|$ is a unit vector normal to the magnetic surface and pointing outwards. For the QHS case in Fig. 8(a), the drift wave spectrum is obtained in the absence of the driving mechanism $\delta=0.0$, indicated by asterisks and for a finite $\delta=0.001$, indicated by squares. Eigenfunctions in different parts of the spectra are shown as insets. The modes are marginally stable in the absence of driving mechanism $\delta$. For finite $\delta$, the weakly and strongly localized modes are driven unstable. At low frequencies the modes are extended along the field line and for higher frequencies they are more localized. This is the case for both QHS and Mirror configurations. The real frequency and the growth rate of the most unstable mode on this field line is found somewhat larger in the QHS case $(6.2312,0.0054)$ than for the Mirror $(5.6122,0.0048)$ configuration. The envelope of the eigenfunction in the QHS case is found to be slightly more localized than the Mirror case. Both the larger value of the growth rate and the localization of the modes along the field line can be understood by examining the local characteristics of the field line in Figs. 5-7.

The effective potential function $U$ of the drift wave equation (left) and the eigenfunctions of the most unstable modes (right) for both geometries are plotted in Fig. 9. $|\mathbf{B}|(\zeta)$ is also superimposed on the eigenfunctions. The modes are localized in the first couple of helical peaks of the potential and become unstable due to finite $\delta=0.001$. However, the tendency for localizing is more evident in the Mirror configuration due to larger peaks in $U(\zeta)$ at $\zeta= \pm 2.5 \mathrm{rad}$.

Figure 10 gives the mode eigenfrequency (both real frequency and growth rate) of the most unstable modes as a function of $b$, other parameters are the same as in Fig. 8. The squares represent the Mirror case and the diamonds the base QHS configuration. It is found that as $b$ increases the growth rate of the modes decrease, which is consistent with earlier tokamak studies. The QHS case has higher growth rates than for the Mirror case. The further difference between the two cases is that the maximum growth rate occurs at $b=0.3$ for the QHS case while it occurs for $b=0.35$ for the Mirror case. This shift toward shorter wavelength for Mirror equilibrium suggests a slight reduction in the transport due to a reduced

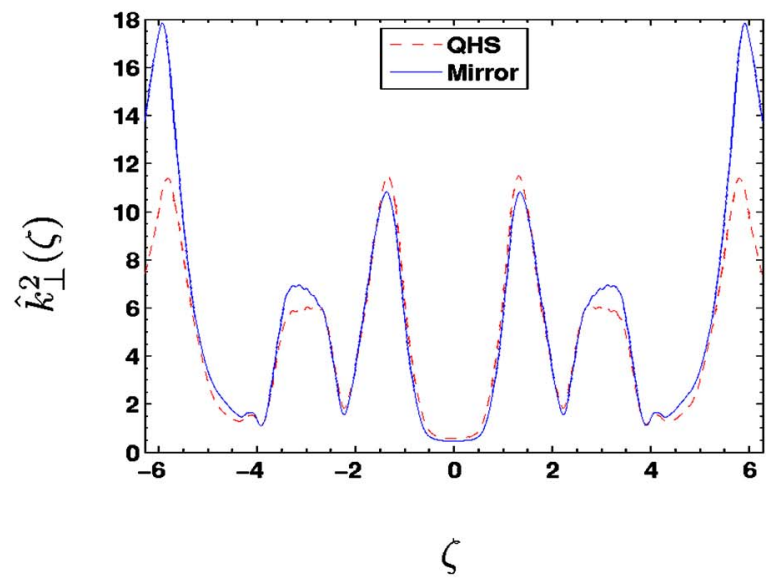

FIG. 7. (Color online). Variation of local magnetic shear $S$ (left) and $\hat{k}_{\perp}^{2}(\zeta)$ (right) as a function of ballooning angle $\zeta$. The other parameters are the same as used in Fig. 5. 


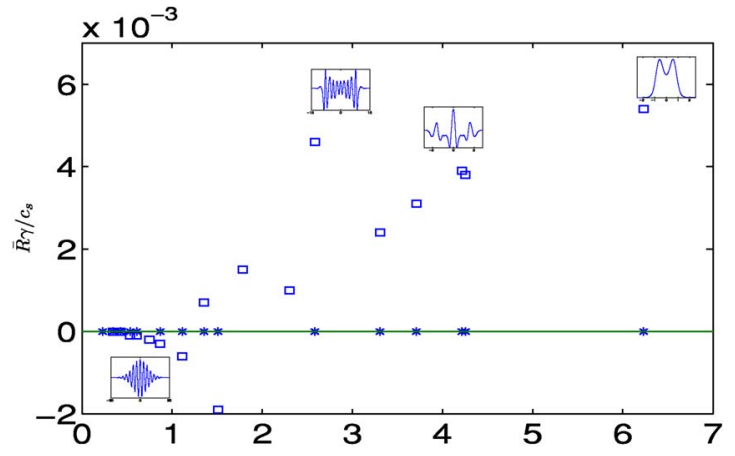

(a) $\bar{R} \omega / c_{s}$

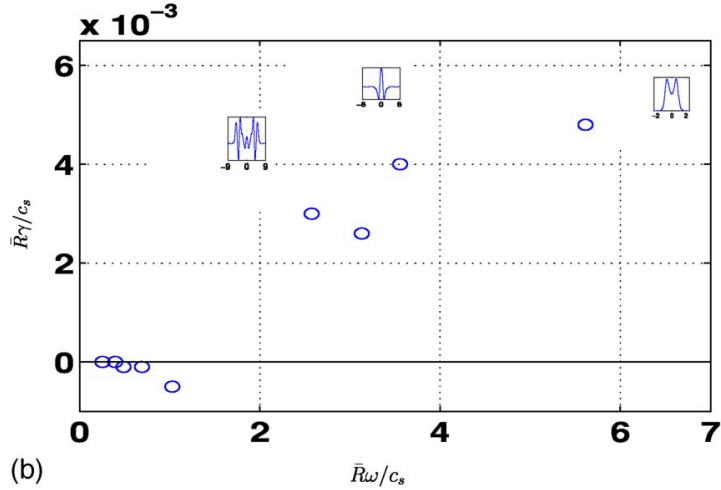

FIG. 8. (Color online). (a) The drift wave spectrum in the QHS case (the normalized growth rate $\bar{R} \gamma / c_{s}$ vs the normalized real frequency $\bar{R} \omega / c_{s}$ ) obtained for $\epsilon_{n}=0.0417, b=0.1, \theta_{k}=0$, along the field line passing through $\theta_{0}=0, \zeta_{0}=0$ on the flux surface $s=0.8980$. Asterisks represent $\delta=0.0$ and the squares represent $\delta=0.001$. (b) The drift wave spectrum (the normalized growth rate $\bar{R} \gamma / c_{s}$ vs the normalized real frequency $\bar{R} \omega / c_{s}$ ) for the Mirror case for $\delta=0.001$. The other parameters are the same as used in (a).

correlation length. However, for higher values of $b$ (shorter wavelengths), a similar magnitude in growth rate is found in both equilibria. As can be seen from the inset eigenfunctions, the modes become more localized along the field line for shorter wavelengths, and feel the similar values of the local magnetic shear along the field line for both equilibria as discussed in connection with Fig. 7 (left). However, for longer wavelengths, modes become extended and modes in the Mirror configurations tend to be influenced by the large negative local magnetic shear; the growth rate decreases more rapidly in the Mirror configuration relative to the QHS configuration.

Figures 11(a) and 11(b) illustrate the variation of the $|\mathbf{B}|$, local magnetic shear, normal curvature, and geodesic curvature, electron drift growth rate, and local shaping term $|\nabla s|$ on the magnetic surface $s=0.8980$ for the QHS and the Mirror configurations, respectively, with all other parameters the same as in Fig. 8. The figures are plotted on the unit cell 0 $\leqslant \theta \leqslant 2 \pi, 0 \leqslant \zeta \leqslant 2 \pi / 4$. The ITG modes in general are important in explaining the anomalous transport in fusion machines but in the present operational regimes of HSX, $T_{e} / T_{i} \gg 1$, and $\eta_{i}<1$. Hence, only electron drift wave growth rates are scanned in Figs. 11(a) and 11(b). Another reason not to scan most unstable ITG modes on the whole surface is that they are more extended than the electron drift modes and are therefore computationally expensive. The highest growth rate for both QHS and Mirror is found in regions where local magnetic shear is small, normal curvature is unfavorable and geodesic curvature and $|\mathbf{B}|$ is minimum. On the other hand, minimum growth rate is found where local magnetic shear is large negative, normal curvature is good, and $|\mathbf{B}|$ is maximum. A large portion of the magnetic surface for both configurations is nearly shearless. The field lines, which cross the region of negative values of local magnetic shear, have the most stable eigenmode. The positive local magnetic shear in the bad curvature region is found to be destabilizing. This is due to the coupling of geodesic curvature and local magnetic shear which modifies the effect of good or bad curvature [cf. Eq. (17)]. Larger $|\nabla s|$ is found at the outer side of the surface of the Mirror equilibrium, which means that the neighboring flux surfaces at the outer side are more compressed in Mirror case than in the QHS. Larger value of $|\nabla s|$ will reduce negative (bad) normal curvature at the outer side of the surfaces and modify the driving curvature effects [cf. Eq. (17)]. This is the reason for finding the lower growth rate in the Mirror configuration than in the QHS case at the outboard points $\left(\theta_{o}=0.0, \zeta_{o}=0.0\right)$ where the curvature is most destabilizing. The calculations suggest that it is useful to
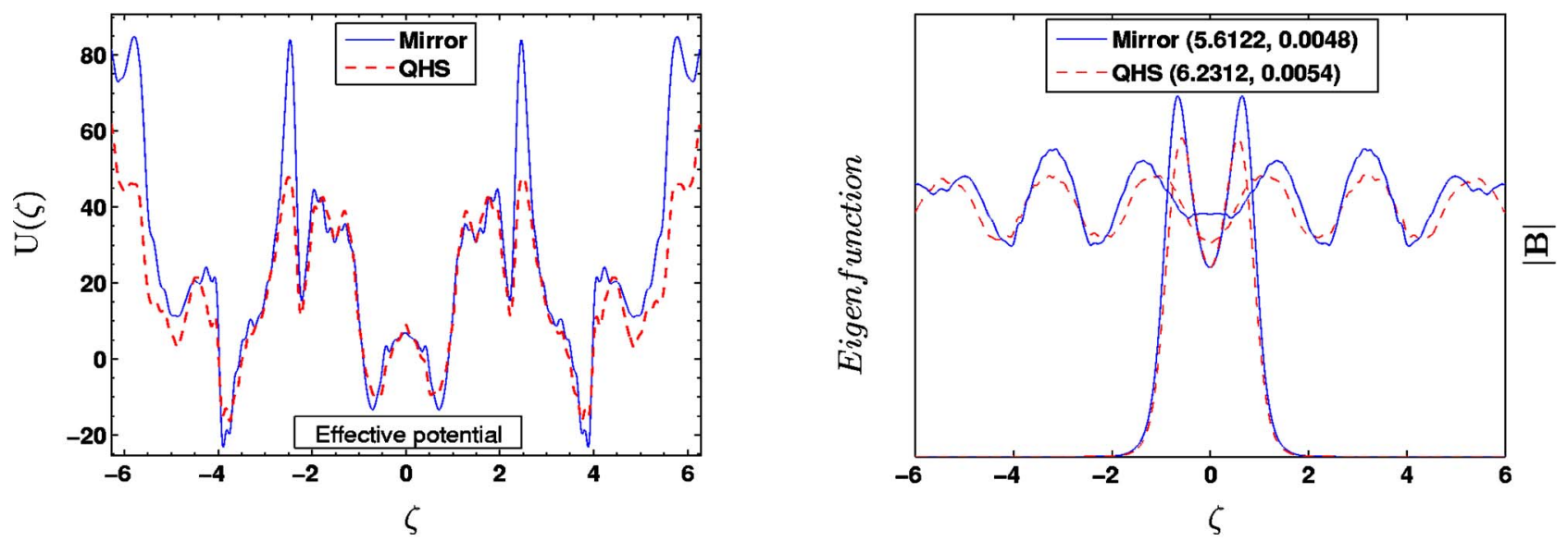

FIG. 9. (Color online). The effective potential, $U(\zeta)$ (left), and the most unstable eigenfunctions along $|\mathbf{B}|$ (right), for the QHS and Mirror case. The other parameters are the same as used in Figs. 8(a) and 8(b). 

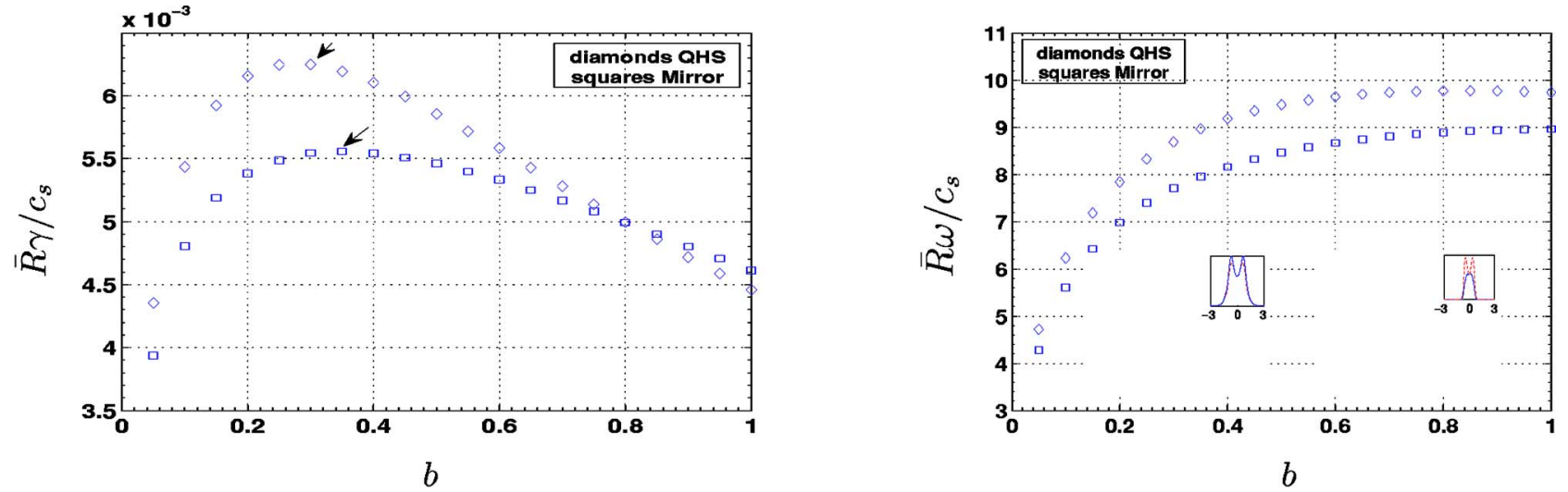

FIG. 10. (Color online). The normalized growth rate, $\bar{R} \gamma / c_{s}$ (left), and the normalized real frequency, $\bar{R} \omega / c_{s}$ (right), as a function of $b$ (squares representing the Mirror case and diamonds the QHS) other parameters are the same as in Figs. 8(a) and 8(b).

compress the magnetic surfaces in regions of unfavorable curvature while making $|\nabla s|$ large in these regions.

A radial scan over the magnetic surface label $s$ is performed for the $i \delta$ and the ITG models for the QHS and the
Mirror equilibria in Fig. 12. In the scan, $b=0.1, \theta_{0}=0, \epsilon_{n}$ $=0.0417, \theta_{k}=0, \delta=0.001$, and $\zeta=0.008$ are kept fixed and for the ITG case $\delta=0, \tau=1.0$, and $\eta_{i}=2.0$, all other parameters are taken to be the same as for the $\delta$ case. It must be re-

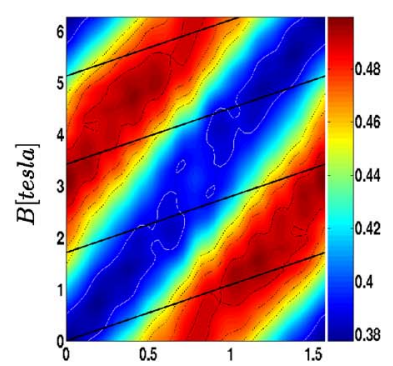

(ai)

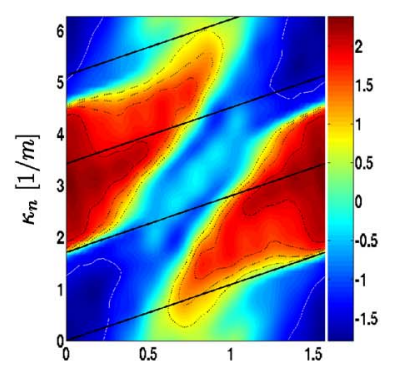

(aii)

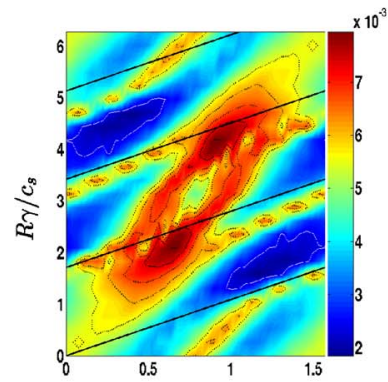

(aiii)
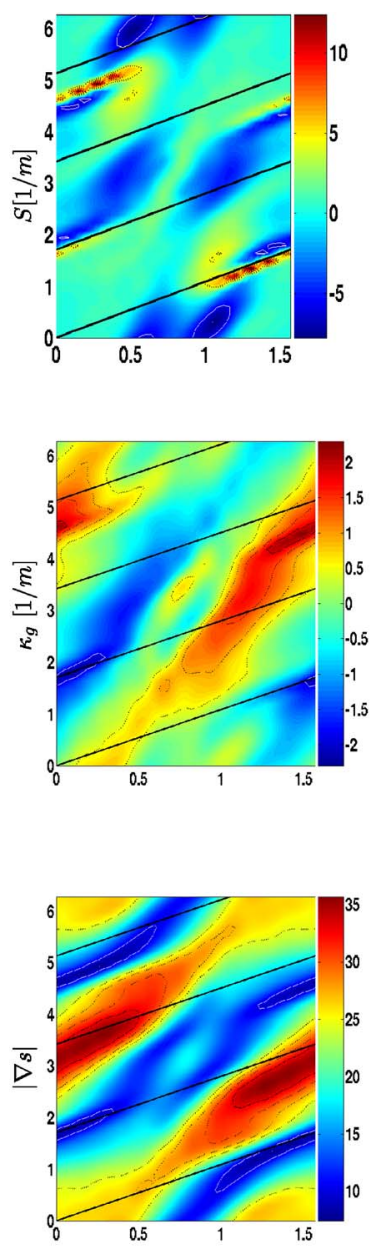

FIG. 11. (Color online). (ai) The $B$ field (left), the local magnetic shear $S$ (right); (aii) the normal curvature (left) and the geodesic curvature (right); (aiii) the normalized growth rate (left) and the local shaping term $|\nabla s|$ (right) on the unit cell in the QHS configuration for the flux surface $s=0.8980$; solid contour line represents the minimum values. Some magnetic field lines $(\alpha=\zeta-q \theta)$ are also superimposed (solid straight lines). (bi) The B field (left), the local magnetic shear S (right); (bii) the normal curvature (left) and the geodesic curvature (right); (biii) The normalized growth rate (left) and the local shaping term $|\nabla s|$ (right) on the unit cell in the Mirror configuration for the flux surface $s=0.8980$; Solid contour line represents the minimum values. Some magnetic field lines $(\alpha=\zeta-q \theta)$ are also superimposed (solid straight lines).
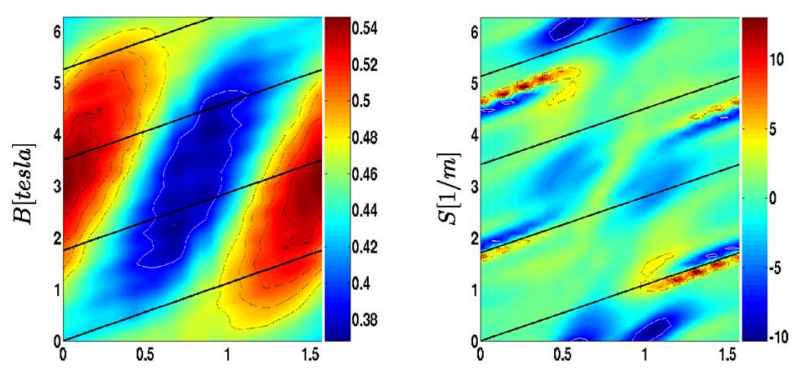

(bi)
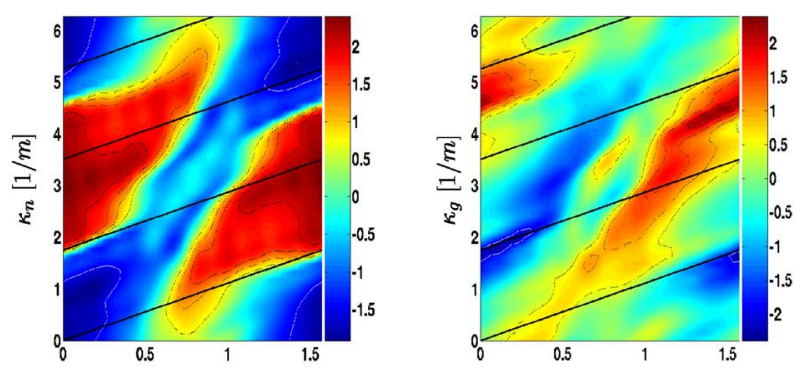

(bii)
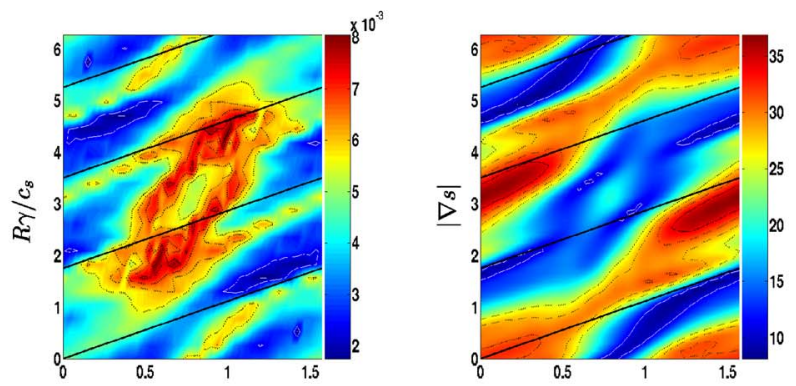

(biii) 

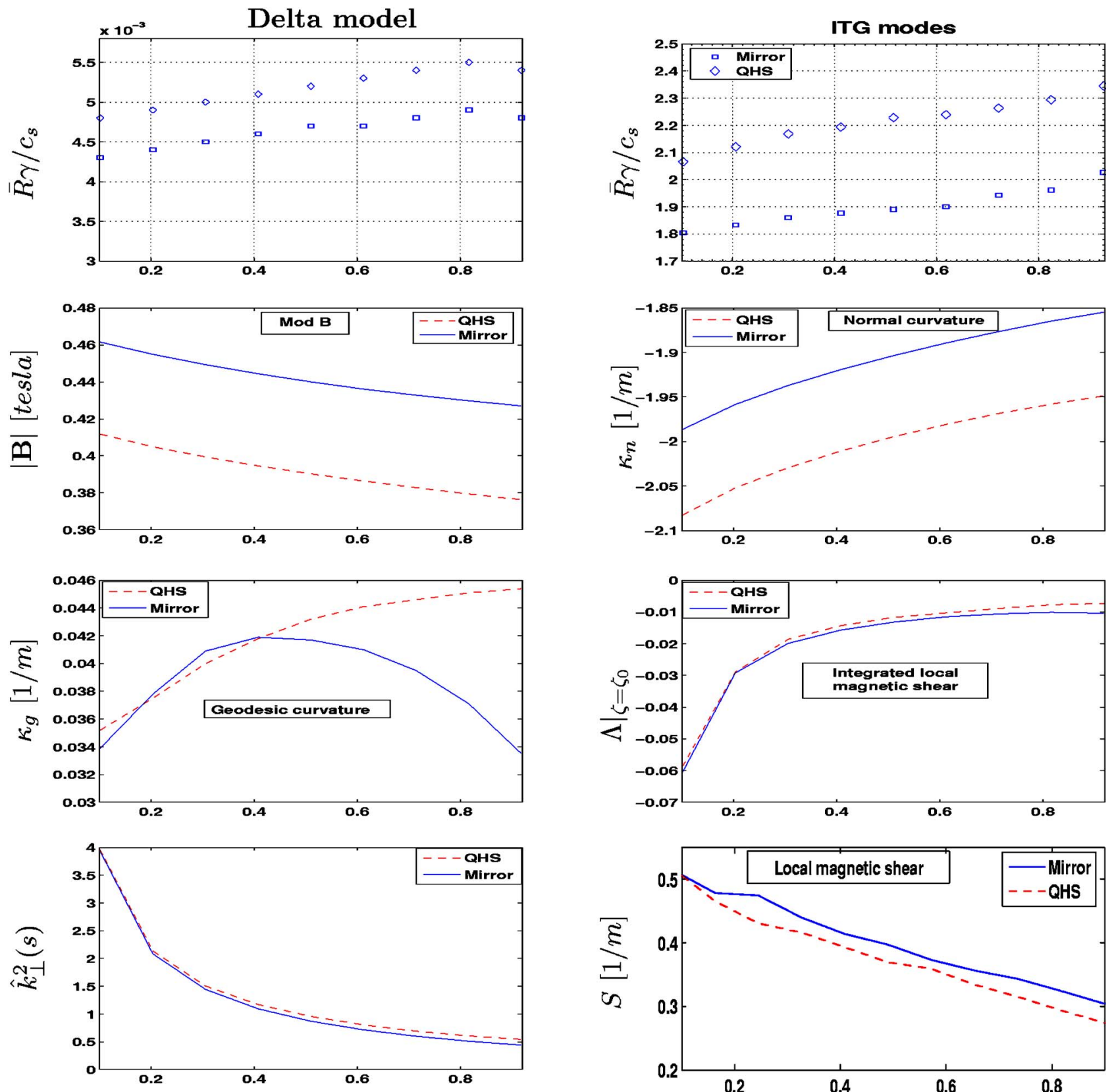

flux label $(s)$ at $\zeta_{o}=0.0125$

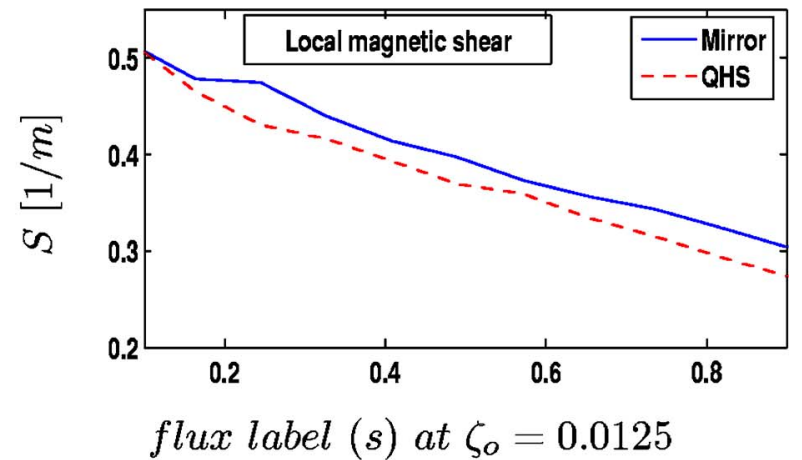

FIG. 12. (Color online). Variation in the $i \delta$ and $\eta_{i}$ model growth rates, $|\mathbf{B}|$, normal curvature $\kappa_{n}$, geodesic curvature $\kappa_{g}, \hat{k}_{\perp}^{2}$, integrated local magnetic shear $\left.\Lambda\right|_{\zeta=\zeta_{0}}$, and local magnetic shear $S$, along the flux label (s) at $\zeta_{0}=0.0125$, other parameters are the same as in Figs. 8(a) and 8(b), while for ITG, $\tau=1.0$, and $\eta_{i}=2.0$. Squares represent the Mirror and diamonds the QHS case.

marked here that $\epsilon_{n}$ is not varying during this scan because our emphasis is to examine the effect of the geometry on the same mode rather than the effects of density profiles. For both models the growth rates are higher in the QHS equilibrium than in the Mirror equilibrium and the growth rate is increasing toward the edge. This is a consequence of the variation of the magnetic filed strength $|\mathbf{B}|(s)$ and $\hat{k}_{\perp}^{2}(s)$, which changes its magnitude from a higher value to a lower value as $(s)$ increases. This is demonstrated by plotting the $|\mathbf{B}|(s)$ and field line bending term as a function of $(s)$. We note that both the integrated local magnetic shear and local magnetic shear are also decreasing with increasing $(s)$. On the other hand, the increase in the growth rate values for the QHS equilibrium can be understood by the normal curvature and $|\mathbf{B}|$ graphs in which QHS has larger unfavorable curvature and small $|\mathbf{B}|$ than the Mirror equilibrium while geodesic curvature alone does not show any pronounced effect on the growth rate.

Figure 13 shows the growth rate and frequency as a function of $\eta_{i}$. We note that in both geometries the mode propagation is in the ion drift direction and the absolute value of real frequency increases from low values at the lower $\eta_{i}$ threshold to around three $\left|\bar{R} \omega / C_{s}\right|$ at $\eta_{i} \cong 2$. However, the level of maximum growth rate is found somewhat 

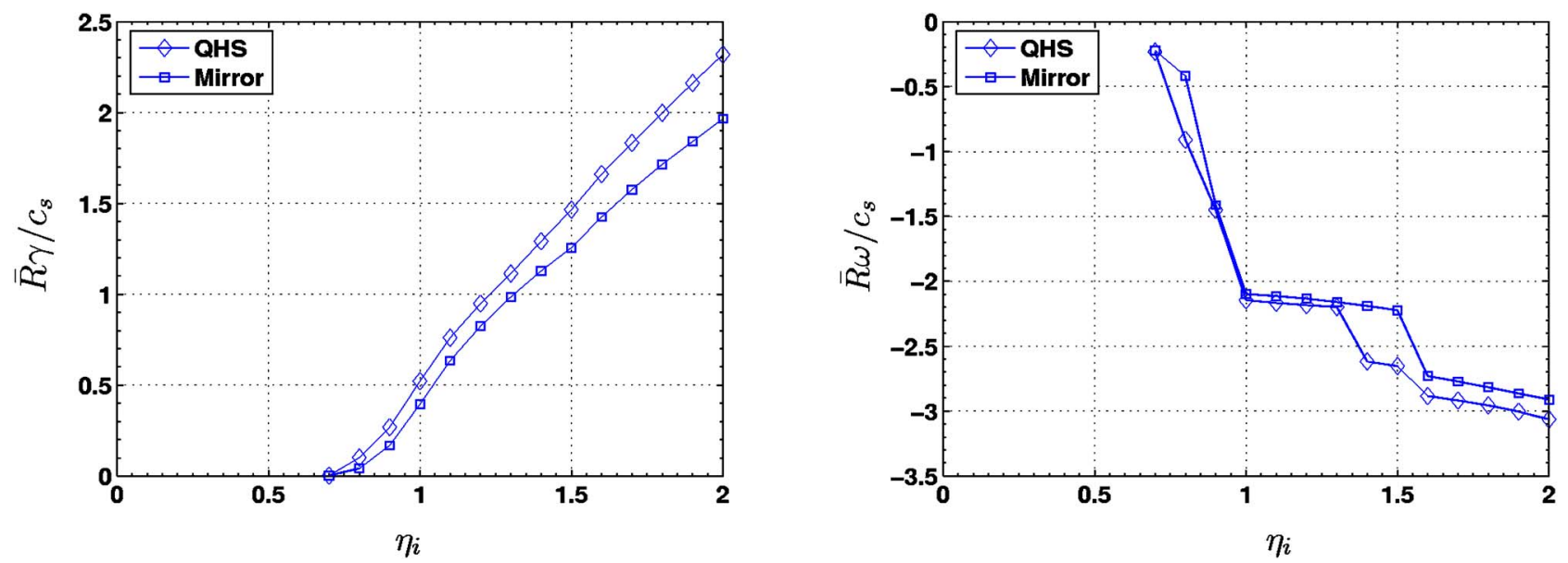

FIG. 13. (Color online). The normalized growth rate, $\bar{R} \gamma / c_{s}$ (left), and the normalized real frequency, $\bar{R} \omega / c_{s}$ (right), as a function of $\eta_{i}$ (squares representing the Mirror case and diamonds the QHS) for $\epsilon_{n}=0.0417, b=0.1, \tau=1.0, \theta_{k}=0$, along the same field line as in Fig. 5.

smaller in the Mirror configuration due to the small magnetic drift frequency. The threshold for instability is approximately $\eta_{i} \cong 2 / 3$.

As one moves in the poloidal direction the equilibrium quantities like the magnetic field, normal and geodesic curvature, and local magnetic shear change periodically. Therefore the spectrum can also vary with changing poloidal angle $\theta_{o}$. This variation is calculated for electron drift and ITG modes in Fig. 14 for the QHS and Mirror configuration. It is found that in the $i \delta$ model, modes are more dependent on the local shear of the magnetic field than on the normal curvature. However, in the ITG model the role of normal curvature is dominant. The maximum growth rate is found at the outboard side where the normal curvature is bad, and the local magnetic shear and geodesic curvature is zero. However, in the Mirror case a noticeable growth rate is also found at the middle point of the surface which lies in the region of favorable curvature. This is due to the low magnetic shear and the large $|\nabla s|$ [see, e.g., Fig 11(a) and 11(c) left], which reduce the good curvature region and modify the driving curvature effects [cf. Eqs. (17) and (18)].

\section{SUMMARY}

A detailed comparison of the linear stability of electron drift modes and ion-temperature-gradient modes (ITG) is made between quasihelically symmetric (QHS) and Mirror mode configurations of the HSX (Helically Symmetric Experiment) toroidal stellarator. The magnet coils in the QHS have been designed so that there is virtually no toroidal curvature in the resulting magnetic field which confines the plasma. In addition to the modular coils that provide the base QHS configuration, there is an additional set of auxiliary coils that allow the neoclassical transport and the stability limit to be independently controlled. Specifically, operating HSX in the Mirror mode configuration increases the neoclassical transport and parallel viscous damping by orders of magnitude (back to the level of conventional stellarators) without appreciable changes in the rotational transform, magnetic well depth, or ideal local MHD stability limit. The influence of plasma geometry on linear drift waves is studied in the QHS configuration and the results are compared and contrasted with the results of a Mirror mode configuration which has high neoclassical transport. The important question discussed here is whether one stellarator geometry is favored over another or if an effective optimization is possible with regard to microinstabilities. The cold ion model used to describe electron drift modes is derived from the fluid dynamics for ions in electrostatic regime whereas electron response is assumed to be close to adiabatic while the ITG model is derived in the short wavelength region by using a two fluid reactive model that includes first order FLR effects as well as parallel ion dynamics and Boltzmann electron. The eigenvalue problem for the drift wave equations is solved numerically using the ballooning mode formalism in fully three-dimensional stellarator geometries. In the cold ion model, the highest frequency modes are strongly localized while the low frequency modes are weakly localized along the field lines. For a finite contribution of $\delta$, the high frequency modes are unstable and the highest frequency and most strongly localized modes have the largest growth rate, while the low frequency modes are damped. It is found that the existence of the high frequency modes and their localization along the field lines are strongly dependent on the local shear and normal curvature of the magnetic field, however, they are less effected by geodesic curvature. Eigenfunctions are found to be more localized in the QHS than the Mirror equilibrium. This is found to be due to the difference in the curvature and the local magnetic shear, which localize the modes along the field lines and constrains them. The minimum growth rate is found in the regions where normal curvature is good and local magnetic shear is large and negative. The large positive local magnetic shear is found to be destabilizing in the bad curvature region. Unstable drift modes are found in both favorable and unfavorable curvature regions. This is partly due to keeping the dissipative mechanism constant. A slight shift toward shorter wavelength is found in the spectrum of the unstable modes for the Mirror case. This shift may tend to reduce the transport by reducing the correlation length in the plasma. Near the axis the growth rate is small and it increases toward the outer surfaces. This can be 

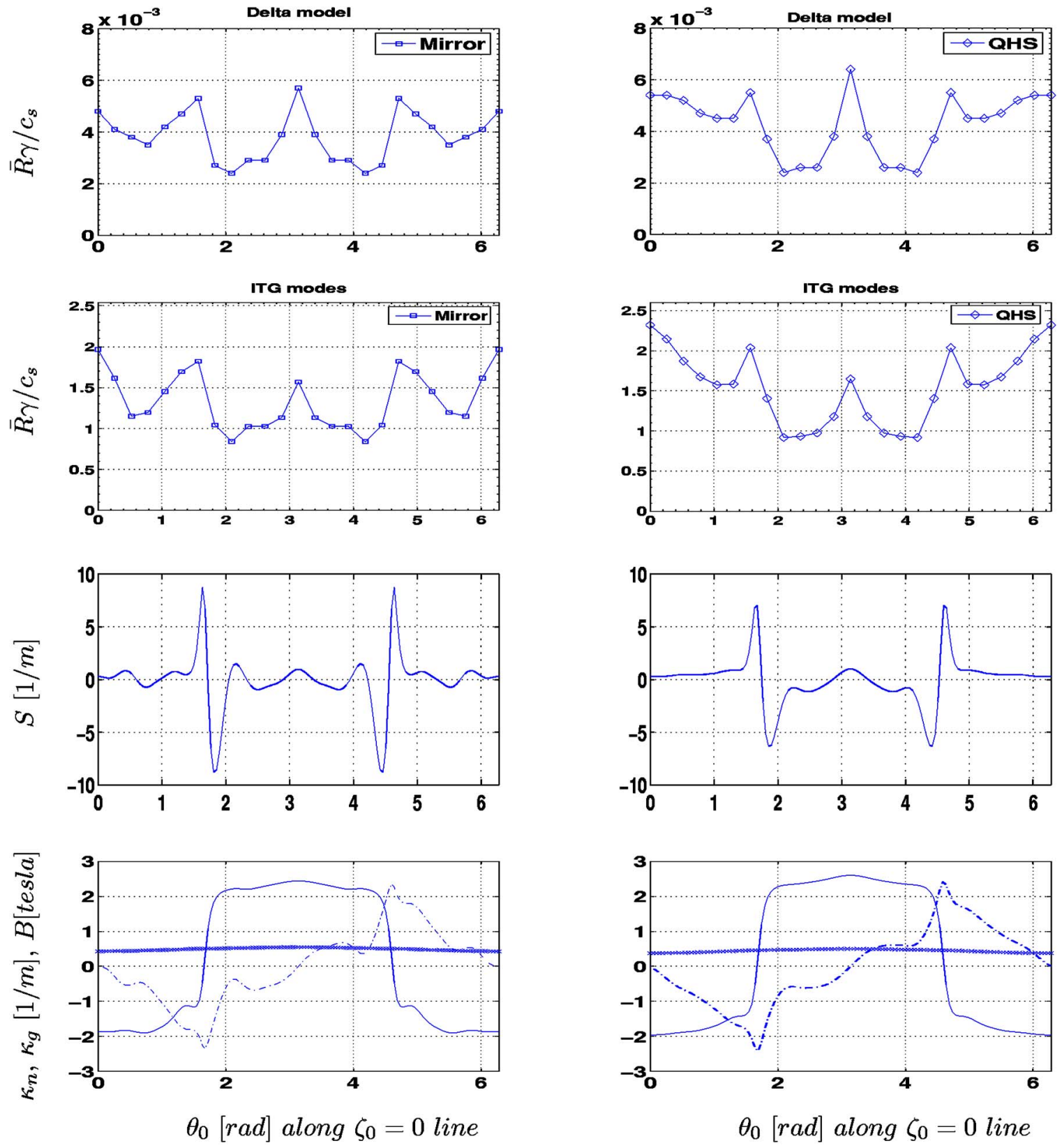

FIG. 14. (Color online). Variation in the growth rate, local magnetic shear $S$, change in field $|\mathbf{B}|$, geodesic curvature $\kappa_{g}$, and the normal curvature $\kappa_{n}$ along $\zeta_{0}=0$ lines in the QHS (left) and Mirror configuration (right). $|\mathbf{B}|$ is the crossed curve $(\times), \kappa_{g}$ is the dashed dotted curve (-.), and $\kappa_{n}$ is the solid curve (-), for $b=0.1, \delta=0.001, \epsilon_{n}=0.0417, \theta_{k}=0.0$ and for the ITG modes $\delta=0.00, \tau=1.0$, and $\eta_{i}=2.0$, on the flux surface $s=0.8980$, other parameters are the same as is used in the $\delta$ model.

due to field line bending $\hat{k}_{\perp}^{2}$, which is large at the center and small at the edge. The smaller growth rate found in the Mirror case is due to small bad curvature and large $|\mathbf{B}|$ in Mirror geometry. An additional effect is due to the large $|\nabla s|$ in the Mirror case, i.e., neighboring flux surfaces at unfavorable region are found more compressed in the Mirror case. The threshold of stability of the ITG modes in terms of $\eta_{i}$ is found to be $2 / 3$ in this fluid model consistent with the smallest threshold for toroidal geometry with adiabatic electrons. Optimization to favorable drift wave stability has small field line curvature, short connection lengths, the proper combination of geodesic curvature and local magnetic shear, large values of local magnetic shear, and the compression of flux surfaces in the unfavorable curvature region. In most of these studies, a simplified adiabatic electrons response is used. Progress on a more rigorous treatment of the electrons will be reported that accounts for toroidally and helically trapped particles and Landau resonances in stellarator geometry.

\section{ACKNOWLEDGMENT}

This research was supported by the U.S. DOE under Grant No. DEFG02-99E54546.

${ }^{1}$ M. Persson, J. L. V. Lewandowski, and H. Nordman, Phys. Plasmas 3, 3720 (1996).

${ }^{2}$ J. L. V. Lewandowski Plasma Phys. Controlled Fusion 37, 1199 (2000). 
${ }^{3}$ A. Kendl and H. Wobig, Phys. Plasmas 6, 4714 (1999).

${ }^{4}$ G. Rewoldt, L-P Ku, W. M. Tang, H. Sugama, N. Nakajima, K. Y. Watanabe, S. Murakami, H. Yamada, and W. A. Cooper, Phys. Plasmas 7, 4942 (2000).

${ }^{5}$ T. Kuroda, H. Sugama, R. Kanno, and M. Okamoto, J. Phys. Soc. Jpn. 69, 2485 (2000).

${ }^{6}$ G. Gost, T. M. Trans, W. A. Cooper, L. Villard, and K. Appert, Phys. Plasmas 8, 3321 (2001).

${ }^{7}$ R. Kleiber, Phys. Plasmas 8, 4090 (2001).

${ }^{8}$ T. Rafiq, R. Kleiber, M. Nadeem, and M. Persson, Phys. Plasmas 9, 4929 (2002).

${ }^{9}$ V. Kornilov, R. Kleiber, and R. Hatzky, Nucl. Fusion 45, 238 (2005).

${ }^{10}$ J. Nührenberg and R. Zille, Phys. Lett. A 129, 113 (1988).

${ }^{11}$ J. Nührenberg and R. Zille, Phys. Lett. A 114, 129 (1986).

${ }^{12}$ M. Nadeem, T. Rafiq, and M. Persson, Phys. Plasmas 9, 1629 (2001).

${ }^{13}$ L. I. Rudakov and R. Z. Sagdeev, Sov. Phys. Dokl. 6, 415 (1961).

${ }^{14} \mathrm{~J}$. Weiland, Collective Modes in Inhomogeneous Plasma (IOP, Bristol, 2000), p. 118

${ }^{15}$ J. Anderson, T. Rafiq, M. Nadeem, and M. Persson, Phys. Plasmas 9, 1629 (2002)

${ }^{16}$ F. S. B. Anderson, A. F. Almagri, D. T. Anderson, P. G. Mathews, J. N. Talmadge, and J. L. Shohet, Fusion Technol. 27, 273 (1995).

${ }^{17}$ J. W. Connor, R. J. Hastie, and J. B. Taylor,Phys. Rev. Lett. 40, 396
(1978).

${ }^{18}$ S. P. Hirshman and O. Betancourt, J. Comput. Phys. 96, 99 (1991).

${ }^{19}$ W. D. D'haeseleer, W. N. G. Hitchon, J. D. Callen, and J. L. Shohet, Flux Coordinates and Magnetic Field Structure (Springer, Berlin, 1991), Chap. 6.

${ }^{20}$ R. L. Dewar and A. H. Glasser, Phys. Fluids 26, 3038 (1983).

${ }^{21}$ A. H. Boozer, Phys. Fluids 25, 520 (1982).

${ }^{22} \mathrm{~J}$. Nührenberg and R. Zille, in Theory of Fusion Plasmas, edited by A. Bondeson, E. Sindoni, and F. Troyon (Editrice Compositori, Varenna, 1987), p. 3.

${ }^{23}$ A. Kendl, Plasma Phys. Controlled Fusion 43, 1559 (2001).

${ }^{24}$ J. M. Greene and M. S. Chance, Nucl. Fusion 21, 453 (1981).

${ }^{25}$ N. Nakajima, Phys. Plasmas 3, 4556 (1996).

${ }^{26}$ R. E. Waltz and R. L. Miller, Phys. Plasmas 6, 4265 (1999).

${ }^{27}$ C. C. Hegna and S. R. Hudson, Phys. Rev. Lett. 87, 035001 (2001).

${ }^{28}$ S. R. Hudson and C. C. Hegna, Phys. Plasmas 10, 4716 (2003).

${ }^{29}$ J. A. Romero, D. Lopez-Bruna, A. Lopez-Fraguas et al., Nucl. Fusion 43, 387 (2003).

${ }^{30}$ G. Y. Antar, G. Counsell, J.-W. Ahn, Y. Yang, M. Price, A. Tabasso, and A. Kirk, Phys. Plasmas 12, 032506 (2005).

${ }^{31}$ T. Rafiq, J. Anderson, M. Nadeem, and M. Persson, Plasma Phys. Controlled Fusion 43, 1363 (2001). 